\title{
Product Design for Reuse of Waste Construction Wood- Teapot Tray Design Practices
}

\author{
Wenming Liu*, Yingjie Cui \\ School of Design and Art, Shenyang Jianzhu University, Shenyang, Liaoning, 110168, China
}

\begin{abstract}
By using waste construction wood as the main raw material, the design practice is based on increasing the storage function of the teapot tray. In the design of the product, we strive to show the unique texture of the waste construction wood. Structural design is based on tenon-and-mortise structure, convenient assembly and disassembly of products as design principles, easy replacement of parts and continuation of product life as design purpose, and teapot tray design through product modular design ideas. Modeling design combined with traditional cultural concepts to "turn waste into treasure."
\end{abstract}

\section{Design basis and ideas}

The design practice of waste building wood product design is a set of product design for teapots and trays. With the improvement of living standards, tea drinking has gradually become a way of life for many people to adjust their mind and body and relieve stress. Statistics show that since 2008, the sales of tea sets have been in the leading position in the tableware industry, and have driven the development of tableware. With the increase in sales of tea wares, the demand for auxiliary functions such as teapot trays is also increasing.

\subsection{Design basis}

Most of the existing teapot tray products are mainly made of metal, ceramics, bamboo, and wood in terms of material selection. Functionally, they are mainly placed with teapots and teacups. However, in applications, metal, ceramics, and other materials have relatively high thermal conductivity. Well, when placing a teapot soaked in tea, it is easy to cause burns to people's limbs; on the other hand, when the tea is poured, the pouring of tea and the high temperature of the teapot are likely to cause water immersion on the surface of the teapot tray, and heat causes uneven deformation of the wooden teapot tray. , Resulting in product failure and material waste. Waste construction wood is better than new wood in terms of shrinkage, compactness and deformation of the material; as a waste material, its recycling is more economical and economical, and the use of materials as a consumable product can greatly reduce the waste of wood; Its material has good heat insulation, practicality, safety and other characteristics; the more important point is that the worm eyes, plaques, knots of waste construction wood have more retro charm than new wood, which not only helps The creation of the teapot tray in the atmosphere, and at the same time, the teapot tray can be placed as a craft by design and processing. [1]

\subsection{Product status and design ideas}

The design of the existing teapot and tray products is mainly based on modeling. It tries to use different interesting designs that are close to people's lives and product design methods that use Chinese culture and symbols. In terms of product use, it can meet the basic needs of users to place teapots, but the functions are relatively single. On the other hand, many incidental items such as tea cup trays, instant tea bags, tea clippers, tasting cups are not well stored. People usually put them in tea boats or store them separately, but the tea boat space is relatively limited Excessive stacking and placing is not only detrimental to the aesthetics of the tea boat, but also occupies space and causes problems such as the inconvenience of using the tea boat. On the other hand, the separate storage of tea sets will also affect the convenience of people. The existing teapot and tray products (Fig1) are mainly based on the design elements of traditional Chinese culture, such as patterns, symbols, characters, landscapes. They are relatively lacking in novelty, and meanwhile, product homogeneity is more serious. The thinness of the volume makes it impossible to display the material texture of the product, and at the same time, the exquisite shape cannot be fully presented to the user. [2]

Therefore, this product design practice takes the functional design of the teapot tray as the starting point, and uses waste construction wood as the main raw material to increase the product's storage function as the starting point. In the appearance of the product, by showing the unique texture of the waste construction wood, this design uses traditional cultural concepts to "turn waste into treasure". The structure is based on the tenon-and-mortise structure as the design basis, the

"Corresponding author's e-mail: 83373734@qq.com 
product's easy assembly and disassembly as the design principle, and the easy replacement of parts and the continuation of the product's service life as the design purpose. The design is based on the modular design concept of the product. The overall design adopts small parts processing and combined design, which is not only conducive to the reuse of waste construction wood, but also can build a durable recycling and processing production line for the reuse of waste construction wood.

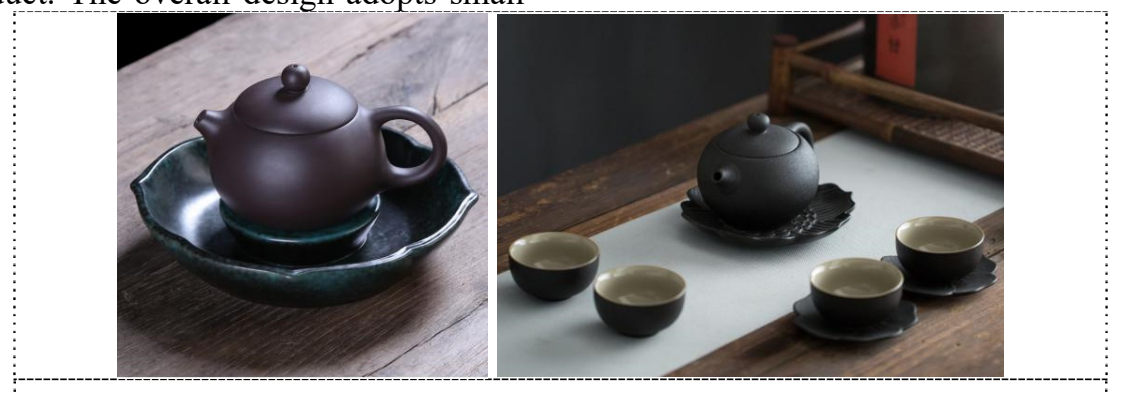

Figure 1. Existing incense stick products.

\section{Scheme design practice}

\subsection{Scheme design one-- "Box" series teapot tray}

Option 1 This teapot tray (Fig2) incorporates the modeling structure of ancient Chinese architecture in its shape. The main body adopts an octagonal structure. The upper cover plane has a convex perfect circle shape, and the upper cover plane is larger than the bottom plane. The base part uses two strips of wood to cross as a base to support the entire pot holder. The lower strips of wood are opened above the center, the upper strips of wood are opened below the center, and the lower strips of wood are used as receiving parts. The trapezoidal opening is used as a connection port with a tenon. An octagonal member is used above the base as a connecting piece connecting the base and the bottom of the tray. The connector and the bottom of the teapot tray use an octagonal plane, and the top is provided with a horse pin tenon. The bottom and the strip of wood are combined with four triangular shapes to cover the two strips of wood. Two of the triangular shapes are trapezoidal at the bottom The mouth is connected with the trapezoidal mouth of the strip of wood below, and the entire teapot tray base is fixed by a tenon connection (Fig3).
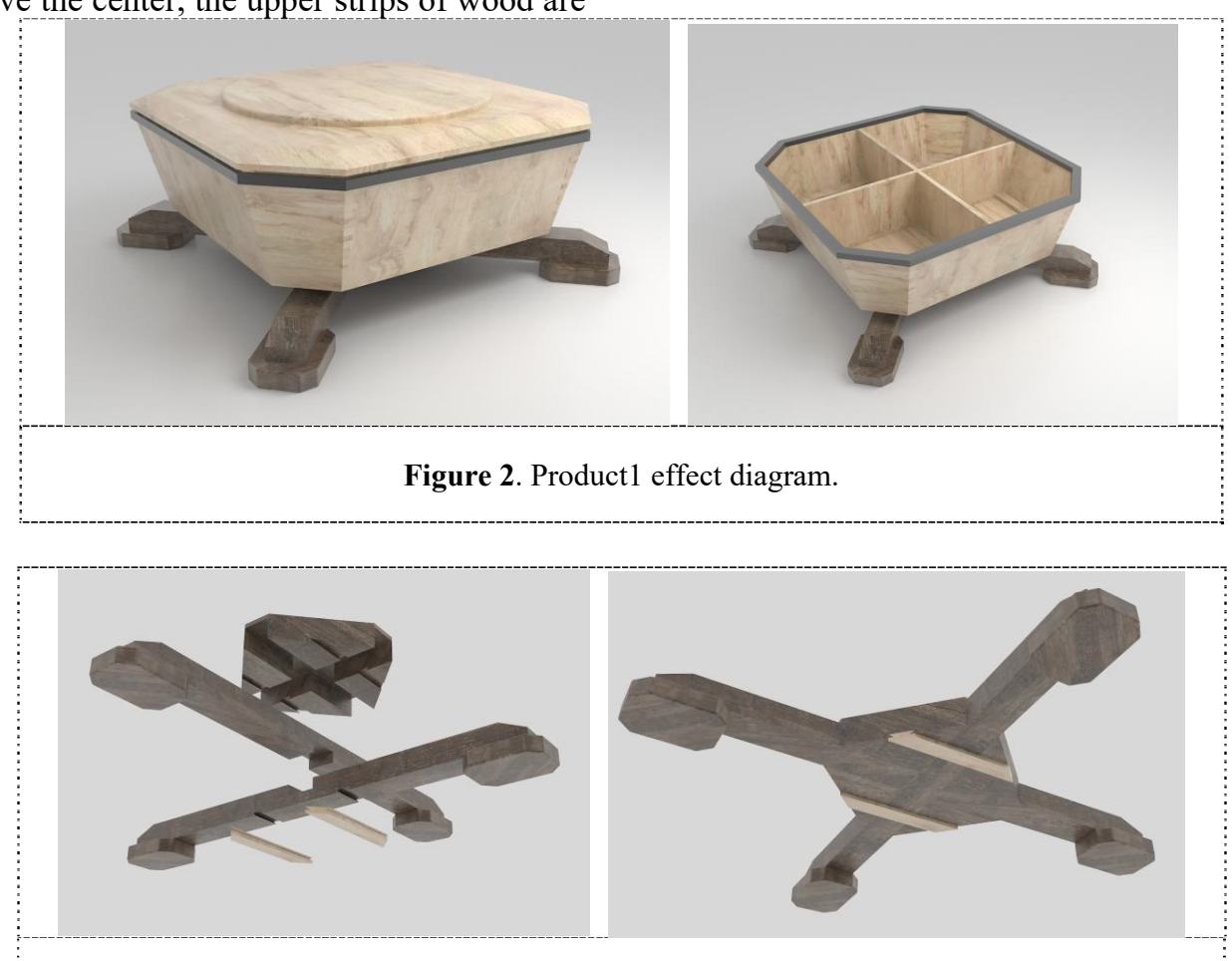

Figure 3. Product 1teapot tray bottom design.

The whole body of the teapot tray adopts the concept of tenon-and-mortise structure and flat tenon-angle combination. Each face of the octagon is divided into modules of equal proportions. The method of joining four long sides and four short sides is used. There are five grooves on the long side and five tenons on the short side. In order to ensure that the main body of the entire teapot tray is firm and difficult to shake after assembly, the connecting part of the bottom plate of the pot holder 
and the base is carefully processed. The top of the bottom plate (Fig4) is opened with a cross-shaped notch to place some partitions in the pot holder box. The

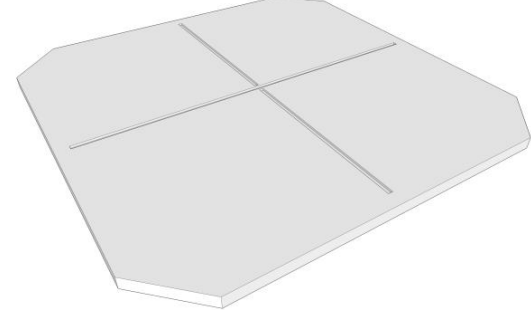

Figure 4. Teapot tray bottom plate top.

The divider of the storage box (Fig6) consists of two trapezoidal plates. The upper trapezoidal plate is slotted from the midpoint of the vertical edge of the plate, and the lower trapezoidal plate is slotted from the midpoint of the vertical edge of the plate. The lower trapezoidal plate has a plug head on each side of the bottom edge, which is used to engage two of the four long side faces of the pot holder to make the entire pot holder structure more stable. Two of the four long side edges of the tray body have a notch corresponding to the insertion

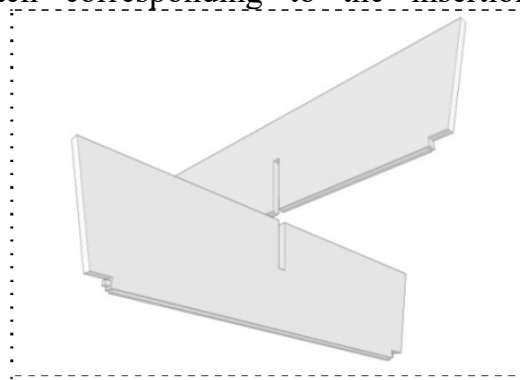

Figure 6. Storage divider. bottom (Fig5) is the groove of the horse pin on the base part. The pot holder base can be connected to the bottom edge of the storage box through the horse pin.

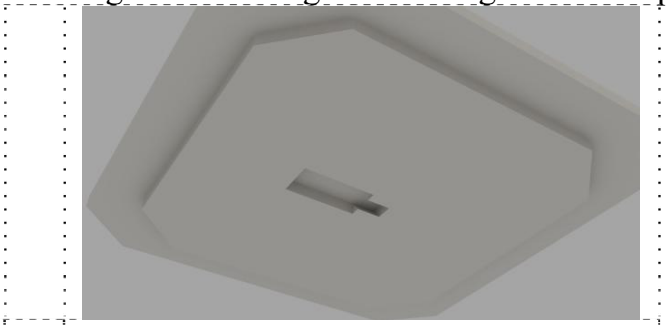

Figure 5. Bottom of teapot tray bottom.

protrusion on both sides of the bottom edge of the trapezoidal plate in the middle and lower part of the partition plate. (Fig7) After the partition plate is installed on the bottom plate (Fig8), the two long face edges of the pot holder are spliced in advance (Fig9), which can ensure the more accurate position of the long face edges of the pot holder to prevent long The unfixed surface causes installation problems caused by displacement, scattering, etc. At the same time, the inner partition structure can be ensured to be stable.

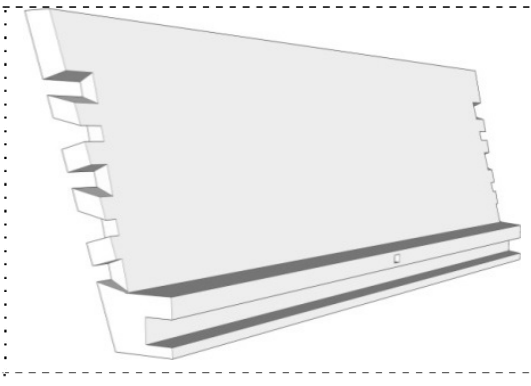

Figure 7. Notches are reserved on the long side of the tray.

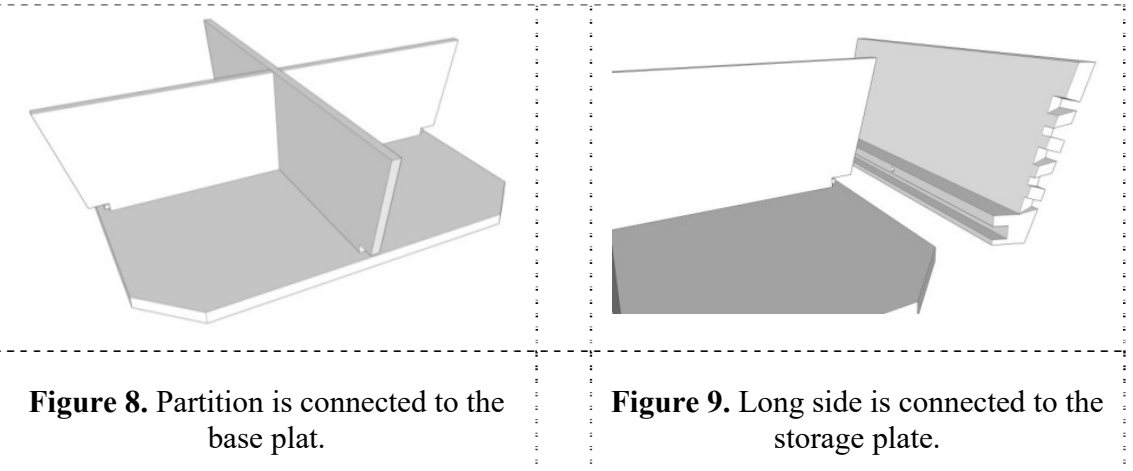

After the installation of the fixed long side, the remaining long side and short side need to be assembled first (Fig10), because the slot design uses an irregular trapezoidal structure, the purpose is to ensure that the combined part will not cause a pot due to displacement. The structure of the support is scattered, so the remaining long and short sides cannot be installed separately. You need to combine one long side and two short sides separately, and then insert the two sets of combined sides into the main structure of the pot support respectively (Fig11). 


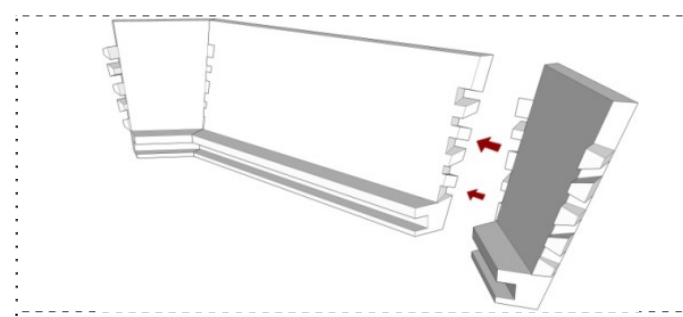

Figure 10. Combination of long side and short side.

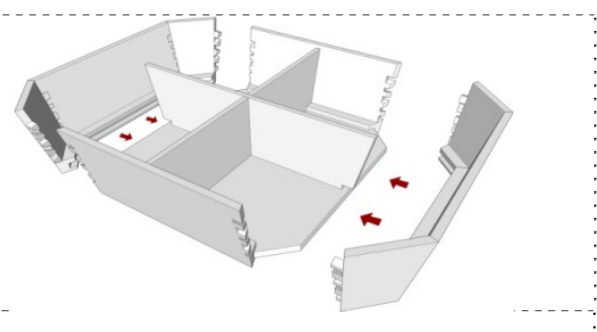

Figure 11. Combination into the main body of tray.
After the installation of the main structure is completed, a metal edge bead is installed at the mouth of the storage box. The first is to ensure that the inserted assembly is stable to avoid loosening due to displacement. The second is to increase the tray closing decoration and at the same time support the tray box cover. A gap is reserved after the lid is installed, which is convenient to remove during use, and it can increase the aesthetics of the top of the pot holder. The straight structure of the base of the pot holder and the tenon are made of waste fir. The other main structures are made of white maple. The length and width of the storage box are $226 \mathrm{~mm}$ each, the height is $77 \mathrm{~mm}$, and the overall height is $104 \mathrm{~mm}$. The overall color is beige and brown. The overall design features are distinctive, and the characteristics of materials such as worm eyes, knots, and plaques of waste construction wood are fully displayed in appearance. The contrast between wood and metal materials highlights a more modern design texture, and it is easier to shape for young people. Accepted, while breaking through the conventional modeling style of traditional boxes, it also contains quaint structures and styles. (Fig12)

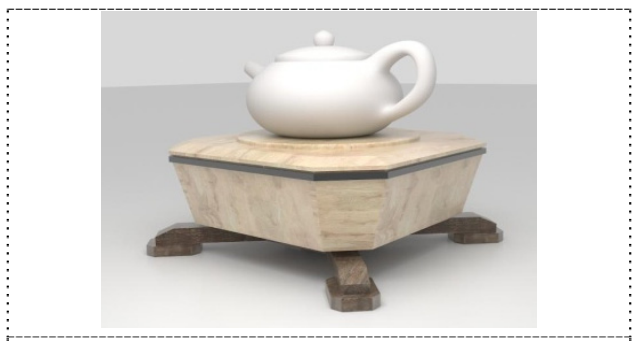

Figure 12. Product 1 effect diagram.

\subsection{Scheme design two-- "Box" series teapot tray}

The overall design of the second teapot tray adopts another relatively simple design style, which is different from the first one. The overall shape and structure of the second embodiment are simple and simple. The entire design is composed of two boxes spliced. The pin is used as a connection method (Fig13). A semi-circular groove is opened at the top of the connection of the box. After being assembled, a perfect circular groove can be formed to place the teapot.

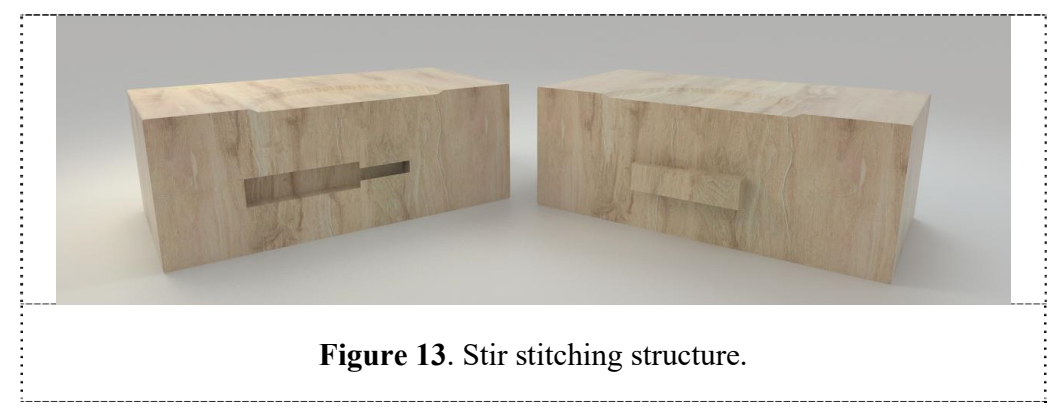

The connection between the handle of the drawer and the door of the drawer still uses a horse pin as the connection (Fig14), because the drawer is used more frequently, and the short side connection end of the pin of the horse pin is weak. The pin head is worn and may even break, so the combination of two handles is used to share the force. On the other hand, in order to ensure that the two handles will not be misaligned and damage the aesthetics and stability of the product, a circular hole is connected at the joint of the two handles as a fixed structure of the two handles.

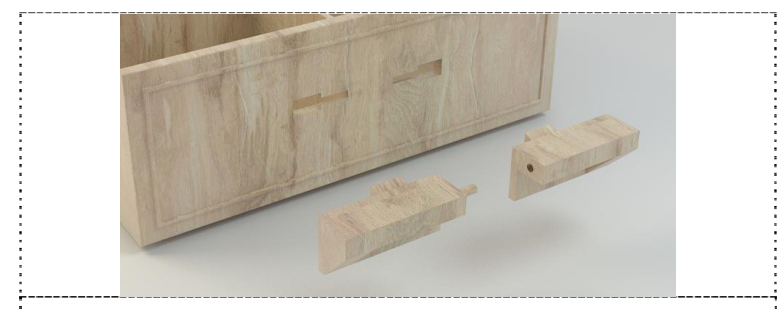

Figure 14. Drawer handle structure diagram.

The storage box adopts a conventional drawer-type drawing structure. Two drawers each have a partition plate inside, and the whole is still four compartments, which are used to hold different tea supplies and 
facilitate the classification of items. The whole scheme is simple and simple in shape. The material is made of waste white maple. The pot holder highlights the texture and texture of the white maple. The simple shape and bright colors give people a fresh visual enjoyment. The block will give the product a sense of precipitation over time, as if this teapot tray has experienced a long history. (Fig15)

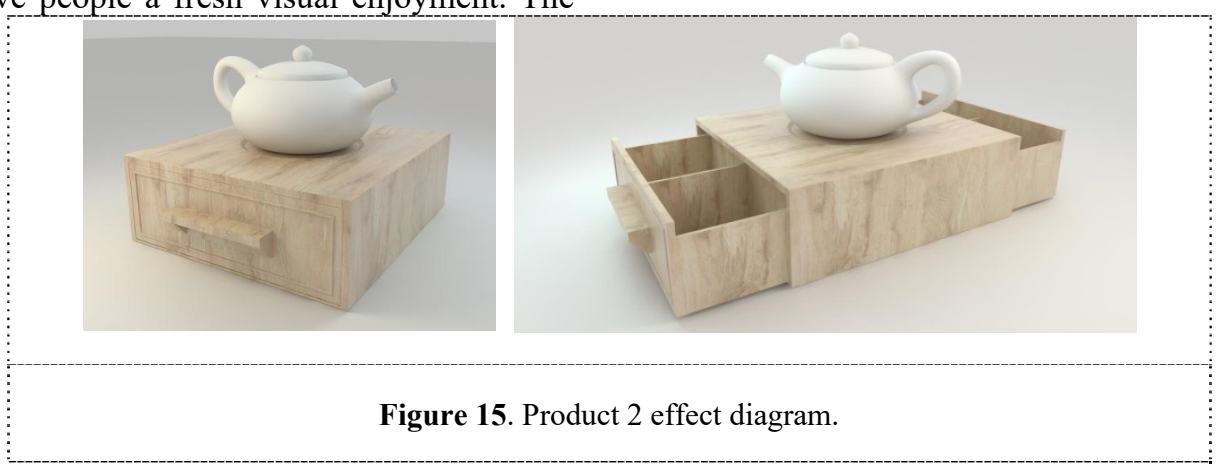

\section{Design plan evaluation}

By using the tenon-and-tenon structure, the reuse design practice of waste construction wood is carried out. In terms of product style, it breaks through the traditional shapes and concepts of traditional teapot trays, and combines product design with functional, replacement, and modular design ideas. In terms of material selection, product design using waste construction wood can fully display the texture of the unique materials such as the texture and knots of the waste construction wood, and it can also give play to the characteristics of the waste construction wood that is not afraid of moisture, difficult to deform, and more stable. Therefore, waste construction wood is very suitable for consumable applications of teapot tray products. By improving the aesthetics, artistry, and value-added properties of waste construction wood through design means, it can increase the aesthetic value of the product and achieve the design goal of "turning waste into treasure". On the other hand, in terms of product safety, it also fully utilizes the application characteristics of wood's poor thermal conductivity, anti-drop, anti-smash, anti-cut and other materials. It is a good application method and approach for the recycling of waste construction wood. This group of products has also passed the examination of the State Intellectual Property Office and obtained a design patent certificate.

\section{Conclusion}

Through the practice of product design, the practice of designing tea tray products using waste construction wood as a raw material combined with metal materials has been conducted in-depth design research in terms of product shape and content, function and replacement. In the design of product shape and connotation, it combines the traditional cultural elements of the "round circle place". In terms of product structural design, it makes more use of modular combinations and tenon-andmortise structure to make the product structure more fully reflect the balance of yin and yang cultural attributes better reflect the artistic display of products and the fun of using products.

\section{References}

1. He, R.K.(2016) History of industrial design. Higher Education Press, Beijing.

2. Michael Burd. (2013) 100 great ideas to change the arts. China Photography Publishing House, Beijing.

3. Wang, S, Q Shen, T. (2015) Japanese design. Guangxi Fine Arts Publishing House, Nanning.

4. Wang.S.X.(2010) Study of Ming Style Furniture. Beijing Sanlian Press, Beijing.

5. He, H, C.(2018) Research on contemporary value of Chinese traditional crafts. University Of Science \& Technology Beijing, Beijing.

6. Kenya Hara.(2017) Japanese design. Guangxi Normal University Press, Guilin. 